\title{
Corpus
}

Archivos virtuales de la alteridad americana

Vol. 9, No 2 | 2019

Julio / Diciembre 2019

\section{La percepción en torno a la música en los informes parroquiales del obispado de Durango (1813-1814)}

The appreciation about music of the parish priests of the Diocese of Durango (1813-1814)

\section{Massimo Gatta}

\section{QpenEdition}

\section{Journals}

Electronic version

URL: http://journals.openedition.org/corpusarchivos/3212

ISSN: 1853-8037

\section{Publisher}

Diego Escolar

\section{Electronic reference}

Massimo Gatta, «La percepción en torno a la música en los informes parroquiales del obispado de Durango (1813-1814) », Corpus [En línea], Vol. 9, No 2 | 2019, Publicado el 26 diciembre 2019, consultado el 27 diciembre 2019. URL : http://journals.openedition.org/corpusarchivos/3212

This text was automatically generated on 28 December 2019. 


\section{La percepción en torno a la música en los informes parroquiales del obispado de Durango (1813-1814)}

The appreciation about music of the parish priests of the Diocese of Durango (1813-1814)

Massimo Gatta

\section{EDITOR'S NOTE}

Fecha de recepción del original: 31/8/2019

Fecha de aceptación para publicación: 19/11/2019

\section{Introducción}

El presente artículo esboza rasgos de la percepción de la música en las parroquias pertenecientes al obispado de Durango en México, en los inicios del siglo XIX.

2 Para ello se ha considerado el discurso en torno a la música contenido en un cuestionario enviado desde las Cortes de Cádiz en 1812 y las respuestas relativas a ese interrogatorio contenidas en los informes parroquiales del obispado neovizcaíno entre 1813 y $1814 .{ }^{1}$ En la actualidad, los espacios que pertenecían a esas parroquias se ubican en los estados de Durango, Chihuahua, Zacatecas y Coahuila de la República Mexicana. ${ }^{2}$ El análisis se articula según cuatro planteamientos.

El primero concierne a aspectos culturales y a su potencial de reescritura de los procesos históricos generales. Tomando en cuenta el corto lapso temporal en el cual se redactaron los informes (entre junio de 1813 y enero de 1814), este ha sido considerado por la historiografía subsumido en las estructuras políticas y económicas relegando el papel de las expresiones culturales en un plano secundario (Bernand, 2014, p. 23). 
El segundo planteamiento se justifica en este vacío historiográfico respecto al caso de Durango en México. De tal manera, este trabajo estudia el fenómeno cultural expresado en y con la música, derivado de la mirada única y diferenciada de un grupo de individuos perteneciente al clero. Esa forma de "ver" se construiría como un proceso epistemológico entre Europa y las Indias cuando la primera imaginó, colonizó y organizó el Nuevo Mundo bajo sus propios modelos y expectativas (Elliot, 2010, p. 255). Sin embargo, habrá que verificar y matizar esa propuesta según una metodología inversa, señalando diferencias y similitudes en ámbitos espaciales y temporales particulares.

El tercero abarca el espacio donde se manifestó esa exploración inédita sobre la música que corresponde a sitios "periféricos", fuera de la cabecera civil y eclesiástica. Las respuestas de los informes parroquiales evidencian la posibilidad de involucrar talantes políticos y sociales presentes en la periferia como clave de complementación, matización e inclusive de reescritura de una realidad histórica céntrica (Burke, 2007, pp. 122-130).

7 El último estriba en una propuesta metodológica interdisciplinaria. Mi reflexión se genera a partir de aspectos que ahondan en la percepción de la música en diálogo con la organología y la filosofía. La descripción de los instrumentos musicales, la manera de tocarlos y el efecto acústico provocado por ellos evidencian costumbres en los grupos socioétnicos que los usaron y ponen al descubierto categorías de juicio estético y aspectos de preparación teórico-musical de los testigos.

8 Por su parte, el discurso sobre la función social de la música de los informes, vinculado a la filosofía política de Platón, enriquece significados en la mirada de la hegemonía según rasgos de continuidad epistemológica.

9 La atención de los investigadores interesados en la temporalidad de crisis para el alcance de la independencia en Durango se ha enfocado en los procesos políticos y económicos en la cabecera neovizcaína. Si bien en ellos no hay la participación de aspectos culturales, parte del contenido de algunas publicaciones brinda algunas claves metodológicas. Miguel Vallebueno (2005) ha analizado la conformación del espacio urbano de Durango a través de una perspectiva demográfica, geográfica, económica y social, desde su fundación en 1563 hasta los primeros años del siglo XXI. Al momento de la crisis de principio del siglo XIX, Vallebueno pone en evidencia la interacción entre la élite urbana, religiosa y civil, entre actitudes de acercamiento y alejamiento que pudieron engendrar expresiones de gustos en el ocio según rasgos novedosos y tradicionales. Indicio de ello fue el surgimiento de la tradición del teatro con la edificación de Teatro Coliseo (1800), en concomitancia con el restablecimiento de la antigua tradición musical de la capilla de música de la catedral (1802).

Otro aspecto clave puesto en evidencia por Vallebueno se manifiesta en la transición estamental a la fase independiente de Durango. Esta se gestaría según condiciones temporales y espaciales amplias: entre ellas el regreso paulatino del gobierno desde el Real de José del Parral, hacia 1760, ausente de la capital desde la primera mitad del siglo XVII; y el auge minero empezado en 1784 por el descubrimiento de la veta mineral de Guarizamey. Por ende, la llegada de nuevos pobladores provenientes de diferentes espacios de la Nueva Vizcaya introduciría cambios de costumbres y de sensibilidad al interior de la vida urbana, no solamente en la novedad sino de aspectos herederos de tradiciones más antiguas. 
11 Angélica Martínez (2013) concuerda con la explicación anterior de Vallebueno, confirmando al inicio del siglo XIX el surgimiento de nuevas sensibilidades al interior del grupo de la clase selecta, definidas por comunicaciones entre ciudad y periferia y que se expresaron según el vestuario, las relaciones interpersonales y las decoraciones internas y externas de los edificios civiles y religiosos.

Por su parte, José de la Cruz Pacheco (2010) resalta la complejidad de la insurgencia de Durango con los movimientos sociales y las relaciones de poder, la participación de individuos pertenecientes a la élite -civil y clerical-y los grupos indígenas en el orden de un espacio que se extendía más allá de la capital de la intendencia. Esa mirada historiográfica deja abierta la discusión sobre cómo esa relación entre grupos socioétnicos se manifestó en la apreciación frente a las distintas expresiones culturales, según cambios y permanencias.

Drew E. Davies (2006) presenta una trayectoria histórica de la capilla de música de la catedral de Durango hasta su disolución en 1840. A partir del mencionado restablecimiento de la capilla en 1802, el tipo de instrumentación y adquisición de partituras impresas según el género instrumental -ejercicios pedagógicos, oberturas, serenatas, valses, marchas, etc. - (Davies, 2013, pp. 21-22) son el indicio de nuevos conductos de comercialización de la cultura musical con centros nacionales e internacionales, así como de gustos sonoros inéditos que se integraron a la devoción. Empero, el musicólogo estadounidense no menciona el contexto de otras realidades sonoras fuera del ámbito catedralicio, ni problematiza la concomitancia de las nuevas tendencias musicales con la práctica antigua del canto llano que seguía vigente en el coro y en las diferentes iglesias parroquiales de la diócesis.

14 A propósito del lugar social del cuestionario gaditano de 1812, es pertinente mencionar cuatro aportes historiográficos.

El primero es una colección de textos a cargo de Francisco de Solano (1988) que trata los cuestionarios españoles enviados a América entre los siglos XVI y XIX. En esta compilación, Pilar Ponce Leiva (1988) capta la estrategia política de los cuestionarios, que fue comprender el espacio geográfico según un sistema político, social y económico para consecuentemente organizarlo (p. XXXI).

16 Perteneciente a la misma recopilación, Antonio Abellán García (1988) comenta que esas formas de reconocimiento geográfico avanzadas por los cuestionarios no fueron inéditas en la óptica de conquista y de gobierno en toda la historia de la humanidad. Sin embargo, dependiendo de su momento histórico, los interrogatorios se perfeccionaron involucrando nuevos temas como el de la demografía, los modos y las costumbres, las lenguas, etc. (p. XXXVII).

Visto el carácter de la compilación de Solano, el contenido específico del cuestionario gaditano de 1812 queda diluido en una visión contextual y no hay seguimiento del contenido de algún corpus de respuestas que haya regresado desde Hispanoamérica.

El segundo es un breve artículo de la historiadora francesa Sylvia Vilar (1971), que se centra en una discusión historiográfica del cuestionario de 1812. La autora califica el documento como "excepcional" por el momento político de crisis de la monarquía española, en el cual surge la novedad en cuanto al tono y su planteamiento relativo a la cultura.

19 El tercer texto, del historiador español Salvador Bernabéu (2010), discute sobre la génesis del cuestionario como instrumento etnográfico, remarcando la importancia de 
considerarlo como "pórtico" de comprensión sobre la otra realidad cultural de los indígenas de Hispanoamérica, con el fin de integrarlos y educarlos como ciudadanos dentro de un nuevo proyecto liberal del imperio. En su reflexión, el autor deja algunas pistas archivísticas interesantes de América y en España.

Los estudios de Bernabéu y de Vilar se centran sobre el cuestionario en sí, en la mentalidad ilustrada que lo originó; mas no reflexionan sobre el impacto de los objetivos políticos originales que se formularon en los informes de los distintos espacios de la Colonia, ni ponderan la problemática del sesgo civil, religioso -o híbrido - de quien circuló el cuestionario, posibilidad que ofrecería la representación de un contexto social, religioso y político específico. Por ejemplo Bernabéu refiere a informes de misioneros, mientras en el caso de Durango el cuestionario fue aplicado por párrocos.

21 En el último y cuarto aporte, Cynthia Quiñones y Miguel Vallebueno (2013) ofrecen el primer relato historiográfico que involucra la música de los informes parroquiales en el contexto de Durango de 1813. Plasmado en un breve subapartado, los autores evidencian cómo la atestiguación de individuos sobre la realidad sonora -junto a la descripción de costumbres, religión, gastronomía y tiempo libre- convergieron en la conformación de un imaginario al final de la etapa virreinal. Según Quiñones y Vallebueno, el sistema documental cuestionario-informes surgiría en base a una "curiosidad ilustrada" (p. 550). Visto de esa manera, no se pone de relieve la función política específica de cada una de esas formas culturales, ni los conductos epistemológicos con los cuales se construyeron.

\section{El contexto político y social duranguense al momento de la introducción del cuestionario gaditano}

Al momento de la llegada del cuestionario gaditano hacia 1813, Durango atravesaba un momento crítico que afectaba tanto las instituciones civiles como las religiosas en las distintas capas sociales, según un contexto general y particular que se explica a continuación.

En términos resumidos, el incremento de la economía novohispana había generado grandes beneficios para la Corona en el ámbito de los grupos europeos vinculados a la explotación de recursos, que se aprovechó de las estrategias de las reformas borbónicas. Por otra parte, los grupos encargados del intercambio de recursos internos en la Nueva España padecieron las limitaciones impuestas por los peninsulares, a saber: impuestos y alcabalas a los productos. Este estado de malcontento condujo a una sublevación general en México en 1810, que se articuló en dos dimensiones: las ciudades y las grandes masas rurales.

Para ese tiempo crítico, Durango como cabecera de la Nueva Vizcaya, alertada por las noticias de conatos revolucionarios, además de ser debilitada constantemente por los ataques de los indios bárbaros (Pacheco, 2010, p. 60), se configuraba como un ambiente socialmente escaldado: la clase hegemónica se conducía de manera errática según sus simpatías y rechazos a la causa realista y había tensiones entre los grupos populares.

Hacia 1808, con la noticia del encarcelamiento del rey Fernando VII, las autoridades locales emprendieron diferentes intervenciones a nivel social, auspiciando la instalación de patrullas y rondas para la tranquilidad interior de la ciudad. Esa 
maniobra contempló la colaboración de toda la población y de las diferentes instituciones como la catedral, que aportaron fondos de sus arcas a través de las gestiones personales del prelado Gabriel de Olivares. ${ }^{3}$

Un momento álgido se manifestó a partir de 1810 cuando, bajo las directivas del gobierno presidido por Bernardo Bonavia y Zapata, gobernador político y militar de la Provincia de la Nueva Vizcaya, se impusieron acciones férreas de represión hacia cualquier individuo o grupos de individuos que manifestasen ideas en contra de la Corona (Vallebueno, 2013, p. 587).

También desde el punto de vista de los grupos socioétnicos, el proceso de crisis que condujo a la independencia puso a Durango en una posición específica en el panorama general nacional, dado que inclusive algunos indígenas, como los tepehuanos, participaron en el conflicto de revolución (Vallebueno, 2013, p. 606). Independientemente de las maneras y de las motivaciones con las cuales ese grupo originario tomó parte en la guerra (milenarismo o manipulación de algunos caciques), esto es indicio de que la crisis de independencia en Durango se conformó como una red de núcleos étnicos que expresaban realidades políticas y culturales más o menos alejadas de la capital.

La Iglesia de Durango, protagonista de esta historia, transitaba por una etapa delicada: después de la muerte del obispo Olivares en 1812, el obispado de Durango se encontró en Sede Vacante, por lo que el Cabildo catedral se encargó del gobierno diocesano hasta 1815.

El 4 de mayo de 1813 llegaba en las manos del deán capitular el cuestionario enviado desde el Supremo Consejo de Regencia. Se encomendó que el interrogatorio circulase por cordillera a todos los ayuntamientos constitucionales, donde debía ser leído tres veces en la iglesia durante la misa mayor, con el fin de que los súbditos supiesen que "las cortes procuraban sostener los derechos y promover la felicidad de los habitantes de la Monarquía". ${ }^{4}$

\section{La música como parte de un proyecto político}

Para los hombres de las cortes gaditanas, la cultura tuvo un vínculo significativo con la pretendida reconstrucción política de España, dado que aquella fue un canal provechoso en el orden del reconocimiento de los espacios sociales del reino.

31 En el caso de las colonias, el reconocimiento de la cultura no podía escapar de plantearse según una "visión indigenista" (Vilar, 1971, pp. 399-404). En ese contexto, el cuestionario de 1812, con sus preguntas de tipo etnográfico, representó un ejemplo significativo de esa estrategia política (Bernabéu Albert, 2010, pp. 38-64).

El cuestionario se articulaba en treinta y seis preguntas sobre diferentes temas: historia, geografía, demografía, relaciones sociales, economía, religión, salud, costumbres y vida cotidiana (Quiñones y Vallebueno, 2013, p. 550). La Pregunta 33 apuntaba a obtener información sobre la música a través del uso de instrumentos musicales, el tipo de sonoridades, su función en las diferentes parroquias de la diócesis, ${ }^{5}$ y se formulaba de la siguiente manera:

Si [los indios] tienen inclinación a la música, qué especie de instrumentos conocen y de cuerda y de viento, si éstos son los mismos que han usado siempre y si conocen los nuestros y los usan. Si poseen algunas canciones en sus idiomas, si son dulces, 
alegres o tristes. Si se inclinan más en esta línea a la música patética o melodiosa o a la guerra, y en caso de usar algunas canciones propias los tonos en que constan y si es posible una exposición y nota de ella. ${ }^{6}$

La interrogación se estructura con un objetivo de reconocimiento hacia el otro a través de una referencia (nosotros), como se revela en el tema de los instrumentos musicales: "si conocen los nuestros [instrumentos] y los usan". ${ }^{7}$ Para la exploración de sonoridades extrañas, los principios estéticos y teóricos de música europea eran los puntos de correlación: "Si [las canciones] son dulces, alegres o tristes"8 o, concerniente a la función social, se cuestionaba una posible relación entre música: "patética o melodiosa o a la guerra". ${ }^{9}$ La pregunta ofrecía la oportunidad de que el cura de alma participara creativamente en la descripción de los cánticos de los indios, según "una exposición y nota de ellos". ${ }^{10}$

En el caso del área periférica del obispado de Durango, los párrocos discutieron, implícita y explícitamente, sobre el poder de la música en el alma humana según un entendimiento universal, reconociendo: "su puesto [sic] que las naciones tengan alguna civilización o gobierno y no sean enteramente bárbaras". ${ }^{11}$ Por ello, la reflexión de los párrocos, aparte de esclarecer el mero fenómeno acústico, se enriquece según el contexto de todos y cada uno de los informes, articulándose con otras percepciones referentes a la realidad demográfica, la situación política, las relaciones sociales, etc. Así las cosas, para muchos observadores de la periferia ya no era posible hablar de una separación nítida entre castas. "Me hace indispensable advertir la confusión que acerca de las castas se encuentra en casi las más familias de la gente de esta población", ${ }^{12}$ contestaba perplejo el párroco de Nombre de Dios, o se criticaba a quien todavía utilizaba "ridículos epítetos de coyotes, lobo y jenízaro". ${ }^{13}$

Y aunque no habrá suficiente espacio de discusión acá, cabe preguntarse sobre las concomitancias entre modos sonoros y las condiciones provocadas por las extensiones espaciales, la orografía, el clima, las variadas formas de medir el tiempo que se comunicaron en el informe y que, de cierta manera, fueron vinculadas a la sonoridad.

No obstante estas diferencias, en esa representación instantánea de la cultura realizada entre 1813 y 1814 en el obispado de Durango, se constató una inclinación general de los indios y de otros grupos socioétnicos a la música: "todas estas gentes son inclinadas a la música", "manifiestan alguna inclinación a la música", ${ }^{15}$ o también "no dejan de ser inclinados a la música". ${ }^{16}$

3 Para los curas, la confusión racial implicaba que la música y las actividades que ella acompañó venían expresadas del "mismo modo" ${ }^{17}$ que los europeos, pero dejaban en evidencia un talante inédito de tipo "americano", como se puede apreciar en la Hacienda de San Diego del Ojo: "las mismas [canciones] que usan los europeos y los españoles americanos". ${ }^{18}$

Entonces, la imagen en torno a la música de la diósecis de Durango describía un ambiente social general, presagio de la futura etapa independiente de México, como también lo aprecia Antonio García de León según un "clima de ruptura de las antiguas barreras étnicas, sociales, de casta, de estamento y prestigio” (2011, p. 17). 


\section{Sonoridad y danza} mirada general presente desde el siglo XVI en América, con el fin de acercarse a la comprensión del fenómeno eliminando su carácter de exotismo. En ese discurso, para nada inocente, el testigo implicaba dos temporalidades: una pasada, de la historia de España, y la otra vigente para la América. A saber, las zambras y leilas importadas por los árabes en la Península durante la Edad Media, siendo análogas a las danzas en el caso de la Nueva España, creaban formas rituales que eran despreciadas por los cristianos (Chalmeta, 1995, p. 17). En los diferentes informes había una apreciación común de la sonoridad de la danza transmitida a través un tono condenatorio. Éste, a su vez, se reforzaba por el contexto general de las parroquias donde los indios eran vistos constantemente como supersticiosos, mentirosos, flojos, promiscuos y ociosos. Sin embargo, para los curas más críticos, la reflexión de dicha actitud corrupta de la feligresía derivaba de un mal gobierno y de una falta de educación escolarizada.

Es posible pensar que, para el momento de crisis social y política del obispado neovizcaíno en 1813, el ritual sonoro del baile fuese percibido como condición para la

Corpus, Vol. 9, No 2 | 2019 
revolución social según violencia y desorden; mensajes simbólicos manifestados por relación de movimientos corporales, intensidades y timbres sonoros extraños que atentaban contra la moralidad, según la idea de: "quien vive el ritual, sufre cambios en su forma de pensar, en su estatus social o en su sexualidad, de forma momentánea o permanente" (Alarcón Guerrero, 2011, p. 38).

\section{El papel social de los instrumentos musicales}

El reconocimiento sobre la sonoridad pretendido en el cuestionario involucró el tema de los instrumentos musicales y, en el vasto espacio del obispado de Durango, los más apreciados fueron la guitarra y el violín: "ni usan otros instrumentos músicos que los corrientes en todo el reino de violín y guitarra". ${ }^{26}$

Empero, en los informes parroquiales, queda la duda de si la guitarra fue sinónimo de vihuela, dado que ambos términos en el corpus de informes se mencionan juntos. En contextos culturalmente heterogéneos de ese tipo, Eloy Cruz (1993, p. 20) acusa una dificultad de definición: más bien el tipo de instrumento correspondería al ámbito social y cultural donde se insertó. En el caso de que vihuela y guitarra fuesen dos instrumentos diferentes, por sus técnicas de ejecución distintas con el "rasgueo" o el "punteo", ${ }^{27}$ ese dato en los informes debería estar justificado por las innumerables aplicaciones de esos instrumentos en los distintos espacios. Y sobre el uso de las dos técnicas en una forma que, según los observadores en la diócesis duranguense, la vihuela y la guitarra se tocaban "del mismo modo que lo usan los españoles". ${ }^{28}$

El párroco de Nieves percibía el protagonismo de la vihuela a través de participaciones solistas: "siendo los cánticos los comunes requintillos". ${ }^{29}$ Si bien los curas no citan el uso del rasgueo, es posible inferir que los feligreses utilizaban esa manera de tocar debido al volumen sonoro necesario para una imposición de la pulsación rítmica y armónica en el fragor de los bailes y convites, que venían acompañados con percusiones y alientos.

49 De haber sobrevivido la vihuela en su tipología "de mano" en los sitios del obispado de Durango desde el siglo XVI hasta los inicios del siglo XIX, su uso representaría la cultura de la gente letrada, en contraste a la guitarra utilizada en el ámbito los grupos populares (Davies, 2009, p. 52).

50 Por la aportación de los curas, el uso de uno o de los dos instrumentos estaría atestiguando la presencia de una tradición sonora representante de una interacción entre culturas pertenecientes a los grupos de élite y populares. Este aspecto se reforzaría por una "guitarra acompañante tanto en la iglesia como en sus bailes y regocijos públicos en la iglesia". ${ }^{30} \mathrm{El}$ papel social y representativo de la guitarra se podría nutrir del estudio iconográfico de los retratos de castas en el contexto novohispano: la guitarra reflejaría una idea utópica de igualitarismo, dado que aparecía en representaciones sin una coherencia: "en cuanto a la casta que tañe la guitarra ni en cuanto a su entorno ni en cuanto a la función del tañedor" (Enríquez, 2009, p. 87).

51 También el violín genera incertidumbres en cuanto a su representación social y cultural, dado que la evidencia documental informa sobre su uso indiferenciado entre los grupos socioétnicos. Eso crea un gran contraste con el uso del violín en el ámbito del repertorio y ejecuciones en las capillas de música, uso que se le dio en las catedrales novohispanas en el siglo XVIII que auspiciaban la devoción según dispositivos estéticos 
sonoros recuperados de los grandes teatros de las grandes ciudades europeas. De todos modos, esa realidad periférica abre nuevos interrogativos sobre la posible utilización del violín aun en los grupos populares del área urbana, visto el pequeño indicio aportado por el cura de San Juan Bautista de Analco, parroquia al límite con la capital: "Digo que la inclinación que se les advierte en la música es tenue, una a que la tiene o uno y otro violín el que la tiene". ${ }^{31}$

Una ulterior discrepancia se verifica desde el punto de vista iconográfico, donde el violín es siempre tocado por un español o un castizo (Enríquez, 2009, pp. 86-87). La observación de los curas de la periferia diocesana sobre el violín es superficial; sin embargo, se podría realizar alguna inferencia para situar el instrumento en su contexto espacial y cultural, por ejemplo, a partir de la tradición laudera que sigue viva en algunas comunidades de las regiones peinadas por el cuestionario. Parece a este punto evidente que, en el vasto espacio episcopal de la diócesis, el violín fue percibido, acompañado por la vihuela y la guitarra, como instrumento ampliamente compartido entre los diferentes grupos socioétnicos, en sociabilidades festivas y religiosas arriba mencionadas.

\section{Un gran paisaje sonoro}

53 Violín, guitarra -o vihuela - y voz humana participaban acoplados en la construcción de la textura sonora de la vida ritual religiosa y profana en el espacio diocesano duranguense. Esta sonoridad, a su vez, se enriquecía con otros instrumentos "altos": pífanos, chiras, chirimías, pitos y las percusiones -estas resumidas por lo general con el término común de tambora-, esbozando el vasto territorio geográfico según un paisaje sonoro, término que designa una realidad social que se codifica según un mecanismo de recepción y apropiación de una sonoridad (Pérez Colman, 2015, p. 107). Con la descripción de los instrumentos y de sus emisiones acústicas, la mirada de los párrocos comprendía la música y la sonoridad con contrastes, referencias y analogías que, como se ha dicho anteriormente, se coligaban a modelos teóricos eurocentristas.

Como resultado, se asomaba una actitud de extrañamiento hacia ciertos fenómenos sonoros: "tocan unos sones en tono extraño que sólo ellos se entienden [...] extraña la música", ${ }^{32} \mathrm{o}$ la imposibilidad de explicación surgía por la falta de preparación teórico musical: "No podré decir porque lo ignoro si tienen o poseen canciones en sus lenguas ni mucho menos, los tonos en que las cantan, porque para esto es necesario tener conocimiento del canto llano o de órgano, del cual carezco". ${ }^{33}$

Esos dilemas sobre la sonoridad tiene justificación en los informes, con el uso indefinido de los términos referentes a la música: tonos, melodías y cánticos, utilizados a menudo como sinónimos. No obstante esas dificultades de aproximación, los párrocos se esforzaron en describir la sonoridad según aspectos patéticos, melodiosos y marciales, enganchándose por ello a un significado político de la sonoridad.

Esta acción comprensiva genera conexión con los contenidos de La República de Platón, obra en la cual se habla de los influjos de melodías y armonías según los aspectos poéticos y morales:

De armonías yo no sé nada; pero déjanos una con la cual se pueda imitar adecuadamente los tonos y modulaciones de la voz de un varón valiente que, participando de un suceso bélico o de un acto cualquiera de violencia, no tiene fortuna, sea porque sufre heridas o cae muerto o experimenta alguna otra clase de 
desgracia; pero que, en cualquiera de esos casos, afronte el infortunio de forma firme y valiente. También piensa en otra armonía con la cual se pueda imitar a quien, por medio de una acción pacífica y no violenta sino atenta de la voluntad del otro, lo intenta persuadir y le suplica [...]. Las armonías que debes dejarnos, pues, son las que mejor imitarán las voces de los infortunados, de los moderados y de los valientes (1988, pp. 170-171 [Rep. III, 399a-b]). una sonoridad "melodiosa y patética", ${ }^{37}$ reflejo de una sociedad que se representó según alegría y sencillez. Mas, en el Pueblo de Santa María de Parras, se percibió "una ausencia de inclinación patética", ${ }^{38} \mathrm{o}$ sea, desentendimiento del significado sonoro $\mathrm{y}$, por lo tanto, la imposibilidad de que este represente algo. En San Juan del Río, hay vestigio de que la música patética se reporta a fronteras políticas y culturales: "patética como en España, melodiosa como en Italia y triste como en América" ${ }^{39}$ Esa constatación se reforzaba con expresiones sobre el sonido del tipo: "es un son muy triste", ${ }^{40}$ en Namiquipa.

61 En el significado de lo patético, los curas de alma del obispado de Durango integraron más apreciaciones según la consonancia y disonancia, lo agradable y desagradable, además de otros ingredientes sensibles.

Por ello, la semántica de lo disonante se conformaba según dos trayectorias explicativas. La primera por la falta de destreza técnico-instrumental, como en el caso de un pífano "mal tocado y en tono de alegría", ${ }^{41}$ o en Santa Cruz del Padre Herrera, lo desagradable derivaba del timbre y la intensidad de los sonidos "lejos de sonar los instrumentos dulces, molestan los oídos". ${ }^{42} \mathrm{El}$ cura de la parroquia de Namiquipa, con más precisión, afirmó que: "sus entonaciones son fastidiosas y todas por un tono, son indemostrables lo uno porque no son permanentes en el canto $y$, lo otro, porque no hay por todo quien hace mínimas o semimínimas, graves ni agudas". ${ }^{43}$

La segunda, para aquellos tonos y cánticos que fueron inexplicables desde el punto de vista teórico, los curas rendían nota de ellos en analogía al tacto y al gusto. Por ejemplo, el cura de Chihuahua informaba que "sus cantos son ásperos y nada melódicos", ${ }^{44} \mathrm{y}$ esta noción de áspero reconduce a la idea de rugoso, o bien, de irregular. Pero en la 
trayectoria de los informes, cabe la posibilidad de que áspero, por su raíz etimológica asper, describa de igual forma el sabor agrio. Es una realidad de gusto ambiguo, entre disonante y lastimero, mas no necesariamente desagradable, que sobrevive hoy en día en el canto cardenche en el área de La Laguna y en los cánticos de los Hermanos de Nuestro Padre Jesús del Templo de San Agustín de la ciudad de Durango (Quiñones y Vallebueno, 2013, p. 575).

\section{Conclusiones}

En referencia al corto lapso de tiempo definido entre 1813 y 1814, el escorzo de la recepción de la música, generado a partir de la atestiguación de los párrocos del obispado de Durango, se ha manifestado según dos talantes.

El primero revela una convicción general de los curas según la cual la música, aunada a las demás expresiones culturales y rituales, cumplía una función política y religiosa en la conformación de un estado, en el cual los indios y los demás grupos sociales podían y debían convivir como ciudadanos.

6 Con el segundo, los curas de alma señalaron el efecto de su posicionamiento frente a otras sonoridades, como aquellas conexas a la danza, a los convites y a las expresiones humanas consideradas como ociosas y pecaminosas. En esas descripciones, aunque ubicadas a principio del siglo XIX, periodo definido generalmente como "ilustrado", emergen los tonos condenatorios de los primeros años de la Conquista. Ese discurso delata la inquietud de que manifestaciones sonoras alternas o desconocidas fueran potencialmente peligrosas para la estructura del imperio.

A propósito de los instrumentos musicales, se ha visto que su función y representación social no coincide necesariamente con las clasificaciones hechas por la musicología, en particular con aquellas realizadas en el ámbito urbano de la episcópolis duranguense. La presencia de guitarra (y/o vihuela) y violín, junto a instrumentos de alientos y de percusión, aportan el indicio de una confusión material y sonora en coherencia con la mixtura racial esbozada en el obispado duranguense. Asimismo, esa gran mezcla de instrumentos y sonoridades fue el vestigio de canales de comunicación cultural entre las diferentes esferas sociales, aspecto que no se ha estudiado al interior de la vida urbana de la capital.

8 Por su parte, el contraste entre la atestiguación de las sensaciones a propósito de tonos y melodías en las parroquias de la diócesis de Durango en los inicios del siglo XIX con los contenidos de La República de Platón permitió entender cómo la música tuvo, además de una función decorativa y de esparcimiento, un rol protagónico de exploración cognitiva y de gobierno.

Finalmente, la consideración de la música en el ámbito de un contexto periférico de Durango ha brindado algunas nuevas lecturas del significado de la música que deberán ser contrastadas con los estudios realizados en el área urbana. De verse identificados y organizados los contenidos de las fuentes de la misma rama presentes en diferentes sitios de América y España, existe una oportunidad invaluable de ampliar el presente experimento historiográfico y conducirlo a planos generales y específicos de mayor envergadura. 
La aplicación de un instrumento de análisis sobre el tema de la apreciación histórica de la música brinda la posibilidad de generar nuevas redes de colaboración entre distintos centros académicos latinoamericanos, norteamericanos y europeos.

\section{BIBLIOGRAPHY}

Fuentes

Archivo Histórico de la Arquidiócesis de Durango (AHAD), Varios, legajo 57, Expediente de los interrogatorios que los párrocos de este obispado van dando absolviendo los treinta y seis artículos del interrogatorio del Supremo consejo de Regencia con fecha del 4 de mayo último se circuló por toda la diócesis en cumplimiento de esta Superior Real Determinación, con el objeto de mejorar la suerte de los indios y formar un sistema más agradable que ha regido, 1813.

AHAD-225 (en microfilm), Correspondencias, Leg. 56, Oficio dirigido por el comandante Nemecio Salcedo al Cabildo Sede Vacante, 1813.

Archivo Histórico del Estado de Durango (AHED), Documentos, oficios y correspondencia sobre la Guerra de Independencia, casillero 4, Exp. 98, 1810, s/f.

"Las canciones y los bailes" en aleph.academica.mx (fecha de consulta 18 de agosto de 2019).

Abellán García, A. (1988). Población y control: las cuestiones demográficas en las relaciones geográficas. En F. de Solano, (Ed.), Cuestionarios para la formación de las relaciones geográficas de Indias, siglos XVI/XIX, (pp. XXXVII-L). Madrid: CSIC.

Alarcón Guerrero, R. (2001). De prodigios y escándalos: música y fiesta o la descripción de lo popular según los escritores del siglo XIX. Antropología. Revista Interdisciplinaria Del INAH, (91), 36-41.

Bernabéu Albert, S. (2010). Las cortes de Cádiz y los indios. En M. M. Ortega Soto, D. Levin Rojo y M. E. Báez-Villaseñor (Coords.), Los grupos nativos del septentrión novohispano ante la Independencia de México, 1810-1847, (pp. 38-64). México: Universidad Autónoma Metropolitana, Unidad Iztapalapa y Universidad Autónoma de Baja California, Instituto de Investigaciones Históricas.

Bernand, C. (2014). Identificaciones: músicas mestizas, músicas populares y contracultura en América (siglos XVI-XIX). Historia Crítica, (54), 21-48.

Burke, P. (2007). Historia y teoría social. Buenos Aires: Amorrortu.

Castellanos, P. (1970). Horizontes de la música precortesiana, Presencia de México. México: Fondo de Cultura Económica.

Chalmeta, P. (1995). Componentes diferenciadores de la cultura andalusí. En P. Chalmeta et al., Cultura y culturas en la historia, (pp. 9-18). Salamanca: Ediciones Universidad de Salamanca.

Cruz, E. (1993). La casa de los once muertos: historia y repertorio de la guitarra. México: Universidad Nacional Autónoma de México, Escuela Nacional de Música. 
Davies, D. E. (2006). The Italianized Frontier: Music at Durango Cathedral, Español Culture, and the Aesthetics of Devotion in Eighteenth-Century New Spain (tesis de doctorado). Universidad de Chicago, Chicago, EEUU.

Davies, D. E. (2009). La armonía de la conversión: ángeles músicos en la arquitectura novohispana. En L. Enríquez (Coord.), Harmonia Mundi: Los instrumentos sonoros en Iberoamérica, siglos XVI al XIX, (pp. 37-64). México: Universidad Autónoma Nacional de México.

Davies, D. E. (2013). Catálogo de la Colección de música del Archivo Histórico de la Arquidiócesis de Durango. Universidad Nacional Autónoma de México, Instituto de Investigaciones Estéticas.

Elliot, J. H. (2010). España, Europa y el Mundo de Ultramar. México: Taurus.

Enríquez Rubio, L. (2009). Entre cuerdas y castañuelas: un vistazo sonoro a la Nueva España galante. En L. Enríquez (Coord.), Harmonia Mundi: Los instrumentos sonoros en Iberoamérica, siglos XVI al XIX, (pp. 64-110). México: Universidad Autónoma Nacional de México.

Eslava Estrada, F. F. (2017). El mundo sonoro de Lucas Alamán: tertulias, jarabes y guitarras de siete órdenes entre el ocaso de la Nueva España y los albores del México independiente. Letras Históricas, 16, 93-110.

García de León, A. (2011). Fandango, el ritual del mundo jarocho a través de los siglos. México: Conaculta.

García de León, A. (2016). El mar de los deseos, el Caribe afroandaluz, historia y contrapunto. México: Fondo de Cultura Económica.

Ginzburg, C. (2011). Occhiacci di legno. Milano: Feltrinelli.

Gombrich, E. H. (2003). La preferencia por lo primitivo. Episodios de la historia del gusto y el arte de occidente. Hong Kong: Phaidon.

Lorente, A. (1672). El porqué de la música, en que se contiene los quatro artes de ella, Canto Llano, Canto de Organo, Contrapunto, y Composicion. Alcalá: Kamares.

Martínez Rodríguez, A. (2013). El momento del Durango barroco. Chapala: Amaroma Ediciones.

Pacheco Rojas, J. de la C. (2010). El proceso de Independencia en Durango. Periodo de la Insurgencia (1808 - 1812). Durango: UJED.

Platón (1988). La República (Conrado Eggers Lan, Trans.). Madrid: Gredos.

Pérez-Colman, C. M. (2015). El campo sonoro y el oído de la sociología: de la doxa sonora al oído sociológico, o los fundamentos teórico-analíticos para el estudio de la vida sonora. Methaodos. revista de ciencias sociales, 3(1), 107-120.

Porras Muñoz, G. (1980). Iglesia y Estado en la Nueva Vizcaya (1562-1821). México: Universidad Nacional Autónoma de México.

Quiñones Martínez, C. y Vallebueno Garcinava, M. (2013). Informe de los párrocos del obispado de Durango sobre la condición de los indios en 1813. En M. Vallebueno Garcinava, (Ed.), Historia de Durango, tomo II, La Nueva Vizcaya, (pp. 548-581). Durango: IIH-UJED.

Ponce Leiva, P. (1988). Los cuestionarios oficiales: ¿un sistema de control de espacio? En F. de Solano (Ed.), Cuestionarios para la formación de las relaciones geográficas de Indias, Siglos XVI/XIX, (pp. XXIX-XXXV). Madrid: Editorial CSIC-CSIC.

Romero, J. L. (2010). Latinoamérica, las ciudades y las ideas. Buenos Aires: Siglo XXI. 
Sánchez Pichardo, P. (2012). Las danzas de pascola y venado. Su cultura material y comportamiento ritual. Anales de Antropología, 46, 135-153.

Vallebueno, M. (2005). Cívitas y Urbs: La conformación del espacio urbano de Durango. Durango: Universidad de Juárez del Estado de Durango, Instituto de Investigaciones Históricas.

Vallebueno, M. (2013). La rebelión de Independencia en Durango. En M. Vallebueno (Ed.), Historia de Durango, tomo II, La Nueva Vizcaya, (pp. 584-611). Durango: IIH-UJED.

Vilar, S. (1971). Une vision indigéniste de l'Amérique en 1812. Trente-six questions élaborées par les Cortes de Cadix. Mélanges de la Casa de Velázquez, 7(1), 399-404.

Zenker, Miguel. “Si los violines hablaran...”. En Museo y memoria: Los objetos cuentan su historia, ICOM México, mayo 2011, Academia Mexicana de Ciencias, Artes, Tecnología y Humanidades, A. C., 2015, disponible en http://www.academia.edu (acceso 2 de junio de 2017).

\section{NOTES}

1. Archivo Histórico de la Arquidiócesis de Durango (AHAD), (en microfilm), Varios, legajo 57. En adelante, Expediente de los interrogatorios de los párrocos.

2. Para los inicios del siglo XIX, el obispado de Durango se ubicaba en el septentrión novohispano y ocupaba un área mucho más vasta comprendiendo el territorio de Nuevo México en los actuales Estados Unidos. Para más informaciones véase Porras Muñoz, 1980, pp. 26-55.

3. Archivo Histórico del Estado de Durango (AHED), casillero 4, Exp. 98, 1810, s/f.

4. AHAD-225 (en microfilm), Correspondencia, Legajo 56, 1813. s/f.

5. Cabe mencionar que, por una parte, las respuestas del cuestionario no abarcaron todas las parroquias del obispado de Durango, condicionado por diferentes casualidades o accidentes en fase de transmisión, como fue el caso de los informes de Sombrerete, Chalchihuites y San Pedro del Gallo. Por otra parte, por la difícil situación política, algunos curas de almas decidieron arbitrariamente no enviar el informe a la capital (Quiñones y Vallebueno, 2013, p. 552).

6. "Pregunta 33", Expediente de los interrogatorios de los párrocos, $\mathrm{s} / \mathrm{f}$.

7. "Pregunta 33", Expediente de los interrogatorios de los párrocos, s/f.

8. "Pregunta 33", Expediente de los interrogatorios de los párrocos, $\mathrm{s} / \mathrm{f}$.

9. "Pregunta 33", Expediente de los interrogatorios de los párrocos, s/f.

10. "Pregunta 33", Expediente de los interrogatorios de los párrocos, s/f.

11. "Reporte de Juan Bautista del Olmo, párroco de San Juan del Río", en Expediente de los interrogatorios de los párrocos, 1813. s/f.

12. "Informe del cura José Miguel de Escontría de Nombre de Dios", en Expediente de los interrogatorios de los párrocos, $1813, \mathrm{~s} / \mathrm{f}$.

13. "Informe del cura Vicente Quiñones del Pueblo de Cinco Señores" en Expediente de los interrogatorios de los párrocos, 1813, s/f.

14. "Informe del cura Rafael Moreno y Lodosa de la Hacienda de San Diego del Ojo", en Expediente de los interrogatorios de los párrocos, 1813, s/f.

15. "Informe del cura Vicente Quiñones del Pueblo de Cinco Señores", en Expediente de los interrogatorios de los párrocos, $1813, \mathrm{~s} / \mathrm{f}$.

16. "Informe del cura José Onofre Oronas de Matachic", en Expediente de los interrogatorios de los párrocos, 1813, s/f.

17. "Informe del cura Francisco Escobar de Namiquipa" en Expediente de los interrogatorios de los párrocos, 1813, s/f.

18. "Informe del cura Rafael Moreno y Lodosa de la Hacienda de San Diego del Ojo", en Expediente de los interrogatorios de los párrocos, 1813, s/f. 
19. “Informe del cura Ramón Galván de San Francisco Xavier de Temosachic”, en Expediente de los interrogatorios de los párrocos, 1813, s/f.

20. "Informe del cura Policarpo Francia de San Francisco del Mezquital", en Expediente de los interrogatorios de los párrocos, 1813, s/f.

21. "Informe del cura Policarpo Francia de San Francisco del Mezquital", en Expediente de los interrogatorios de los párrocos, 1813, s/f.

22. "Informe del cura José Eulalio Rueda" [no se especifica lugar en el informe], en Expediente de los interrogatorios de los párrocos, 1813, s/f.

23. "Informe del cura Rafael Díaz de Noriega de San Miguel del Mezquital", en Expediente de los interrogatorios de los párrocos, 1813, s/f.

24. "Informe del cura José Eustaquio Murguía de San Andrés del Teúl", en Expediente de los interrogatorios de los párrocos, 1813, s/f.

25. "Informe del cura Juan Bautista del Olmo de San Juan del Río", en Expediente de los interrogatorios de los párrocos, 1813, s/f.

26. "Informe del cura Juan Bautista del Olmo de San Juan del Río", en Expediente de los interrogatorios de los párrocos, 1813, s/f.

27. Para más información sobre técnicas de punteo y rasgueo de la guitarra en el contexto afroandalúz en el Caribe véase García de León, 2016, pp. 125-126.

28. "Informe del cura Rafael Díaz de Noriega de San Miguel del Mezquital”, en Expediente de los interrogatorios de los párrocos, 1813, s/f.

29. "Informe de Gabriel Minjares Solórzano, párroco de Nieves, Zacatecas", en Expediente de los interrogatorios de los párrocos, 1813, s/f.

30. "Informe de Rafael Díaz de Noriega, párroco de San Miguel del Mezquital", en Expediente de los interrogatorios de los párrocos, 1813, s/f.

31. "Informe de José Francisco Mijares párroco de San Juan Bautista de Analco", en Expediente de los interrogatorios de los párrocos, 1813, s/f.

32. "Informe de Ramón Galván, párroco de San Francisco Xavier de Temosachic", en Expediente de los interrogatorios de los párrocos, 1813, s/f.

33. "Informe del cura Juan Bautista del Olmo de San Juan del Río" en Expediente de los interrogatorios de los párrocos, 1813, s/f. El canto llano se refiere al canto monódico, mientras el canto de órgano, al canto polifónico, y junto a la composición y al contrapunto conformaban la música (Lorente, 1672).

34. En este sentido, habría una función similar entre los párrocos y los Guardianes del Estado, individuos garantes de la misión del arte y el bienestar de la sociedad (Gombrich, 2003, pp. 11-15). 35. "Reporte de Evaristo Florentino, párroco de San Diego de Canatlán", en Expediente de los interrogatorios de los párrocos, 1813, s/f.

36. "Informe del cura Policarpo Francia de San Francisco del Mezquital", en Expediente de los interrogatorios de los párrocos, 1813, s/f.

37. "Informe de Gabriel Minjares Solórzano de Nieves, Zacatecas", en Expediente de los interrogatorios de los párrocos, $1813, \mathrm{~s} / \mathrm{f}$.

38. "Informe del cura José Nicolás Flores de Santa María de las Parras", en Expediente de los interrogatorios de los párrocos, 1813, s/f.

39. "Informe del cura Francisco Escobar de Namiquipa", en Expediente de los interrogatorios de los párrocos, 1813, s/f.

40. "Informe del cura Francisco Escobar de Namiquipa", en Expediente de los interrogatorios de los párrocos, 1813, s/f.

41. "Informe de Rafael Díaz de Noriega, párroco de San Miguel del Mezquital", en Expediente de los interrogatorios que los párrocos, 1813 , s/f.

42. "Informe del cura José María Castro de Temeyechi", en Expediente de los interrogatorios de los párrocos, $1813, \mathrm{~s} / \mathrm{f}$. 
43. "Informe del cura Francisco Escobar de Namiquipa", en Expediente de los interrogatorios de los párrocos, 1813, s/f.

44. "Informe de José Miguel Salas Valdez, párroco de Chihuahua", en Expediente de los informes de los párrocos, $1813, \mathrm{~s} / \mathrm{f}$.

\section{ABSTRACTS}

This article attempts to recreate the cultural appreciation about music that parish priests of the Diocese of Durango had at the beginning of the 19th century. This is done through the study of the sound culture of different societies located in Northern New Spain, obtained as a result of the analysis of the social and cultural role of sound instruments in dialogue with philosophy and musicology. From this perspective, it is possible to understand unprecedented aspects of meaning and sound function during the final stages of the colonial period. The article concludes that the aforementioned approach to sound culture provides a new insight into the changes and continuities in ecclesiastical mentality during the process of the Independence Revolution, as well as evidences of a shared musical culture between ethnical groups and a sound appreciation that could complement or contrast with the music history of Mexico and Hispanic America.

Este artículo esboza la apreciación de la música en base a la atestiguación de los párrocos del obispado de Durango en los inicios del siglo XIX. A través del estudio de una cultura sonora de sociedades ubicadas en el septentrión novohispano y el análisis del papel social y cultural de los instrumentos sonoros, en diálogo con elementos recuperados de la filosofía y de la musicología, se propone una comprensión alternativa y periférica del periodo histórico correspondiente a las etapas finales de la colonia. A partir de esta aproximación a la historia, se proponen algunas reflexiones inéditas sobre cambios y continuidades en la postura política del clero durante el proceso de la Revolución de Independencia, además de enseñar la presencia de una cultura musical compartida entre los grupos socioétnicos y un gusto sonoro que puede ser de complemento y contraste para los estudios sobre la historia de la música de México e Hispanoamérica.

\section{INDEX}

Palabras claves: Música, política, párrocos, grupos socioétnicos, Durango

Keywords: Music, politics, parish priests, ethnic groups, Durango

\section{AUTHOR}

\section{MASSIMO GATTA}

Escuela Superior de Música, Universidad Juárez del Estado de Durango, México

Correo electrónico: gatta.massimo@gmail.com 